\title{
DETC99/DAC-8640
}

\section{CONTROL BARRIERS OF POSITIONING MANIPULATORS}

\author{
K ABDEL-MALEK \\ Department of Mechanical Engineering, \\ The University of lowa, lowa City, IA 52242 \\ Tel. (319) 335-5676 \\ amalek@engineering.uiowa.edu
}

\author{
HARN-JOU YEH \\ Microtek International \\ Taiwan \\ hjyeh@microtek.com.tw
}

\author{
MOHAMAD ALKAM \\ Jordan University of Science and Technology \\ Amman, Jordan \\ mkalkam@mailcity.com
}

\begin{abstract}
Broadly applicable formulations for identifying control difficulties in positioning serial manipulators are developed based on the Implicit Function Theorem and acceleration analyses on singular surfaces. As a result, voids in the workspace are also identified. Rank deficiency conditions are applied to the positioning Jacobian with joint constraints included in the formulation. Geometric entities upon which the manipulator exhibits control difficulties are identified. These difficulties are associated with a admissible motion along a vector normal to the singular geometric entities. Definiteness properties of a quadratic form that is as a result of normal acceleration define control difficulties. Numerical examples are illustrated.
\end{abstract}

\section{INTRODUCTION}

Control barriers are those surfaces inside the workspace upon which a difficulty in the manipulator's output control is observed. These barriers are associated with restrictions on the output motion control of the end-effector. The purpose of this paper is to analytically identify barriers to output control of serial manipulators, conditions under which difficulties may occur, and to identify voids in the workspace.

Singular behavior of manipulators has been addressed by many researchers Wang and Waldron (1987) and Waldron, et al. (1985), stated that as the Jacobian of the manipulator becomes singular, its columns, which are screw quantities, do not span the full rank of the matrix, therefore, the Jacobian rank decreases by at least one. The proof of the null-space criteria applied to the Jacobian was presented by Spanos and Kohli (1985).

Methods based on Jacobian singularity are found in (Shu et al. 1986, Litvin et al. 1986a, and 1986b). An enumeration of singular sets was presented by Lipkin and Pohl (1991). Shamir (1990) provided tools to determine avoidable singularities for 3-DOF manipulators. Geometric approaches to the study of singular configurations of a manipulator arm were addressed by Lai and Yang (1986) and Ahmad and Luo (1988). A general method for determining the manipulator's singularities was presented by Tourassis and Ang (1992). Pai and Leu (1992) report a characterization of orientation singularities of manipulator arms based upon a mathematical singularity theory. Others that have discussed singularities include Soylu and Duffy (1988), Lai and Yang (1984), Mohammadi et al. (1995), and Merlet (1989).

Early studies that have addressed difficulties in the control of manipulators due to the appearance of interior curves and surfaces (barriers to control) were reported by Nielsen et al. (1991) and Waldron (1987). The fundamental concept of crossable and noncrossable surfaces inside a manipulator's workspace was addressed by Oblak and Kohli (1988).

Haug et al. (1995) presented a numerical algorithm for identifying and analyzing barriers to output control of manipulators using first- and second-order Taylor approximations of the output in selected directions. Haug et al. showed that the output velocity in the direction normal to such curves and surfaces must be zero (Haug et al. 1996). More recently, a criterion to define possible motion (so-called 
feasible trajectory) from a singularity was presented by Chevallereau (1996). A recent comparison of numerical and analytical methods for workspace generation can be found in Abdel-Malek et al. (1997).

In this paper, singular curves and surfaces, inside and outside the workspace, are analytically determined. The background for this method is introduced in Sec. 2. Singular sets used in the identification of barriers of the manipulator's end-effector are developed in Sec. 3. The formulation for determining admissible motion in the normal direction is presented in Sec. 4, while criteria for crossing a barrier and for void identification are developed in Sec. 5. The formulation is verified through examples of planar and spatial manipulators.

\section{BACKGROUND}

A kinematic map of a manipulator $\mathbf{k} \rightarrow K$ is a transformation that maps a configuration $\mathbf{q}=\left[q_{1}, q_{2}, \ldots, q_{n}\right]^{T}$ of an $n$-DOF manipulator to the position of the end-effector reference frame. The position vector of a point on the end-effector of a serial manipulator written as a multiplication of rotation matrices and position vectors can be expressed by

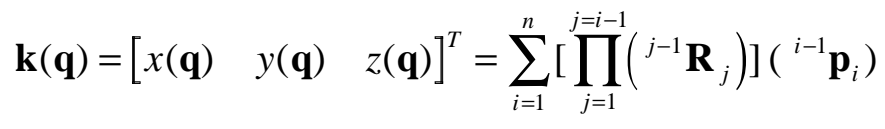

where both ${ }^{i} \mathbf{p}_{j}$ and ${ }^{i} \mathbf{R}_{j}$ are defined using the D-H representation method ( $\mathrm{Fu}$, et al. 1987). Inequality constraints such as $q_{i}{ }^{\mathrm{min}} \leq q_{i} \leq q_{i}{ }^{\mathrm{max}} ; i=1, \ldots n$ are transformed to an equation by introducing a new set of generalized coordinates $\lambda_{i}$ such that $q_{i}=c_{i}+b_{i} \sin \lambda_{i} i=1, \ldots n$,

where $c_{i}=\left(q_{i}^{\max }+q_{i}^{\min }\right) / 2$ and $b_{i}=\left(q_{i}{ }^{\max }-q_{i}{ }^{\min }\right) / 2$ are the mid-point and half-range, respectively. For an assembled configuration at $\mathbf{p}^{E}$, a vector constraint function is developed

$$
\boldsymbol{\Phi}\left(\mathbf{q}^{*}\right)=\left[\begin{array}{c}
\mathbf{k}(\mathbf{q})-\mathbf{p}^{E} \\
q_{1}-c_{1}-b_{1} \sin \lambda_{1} \\
\vdots \\
q_{n}-c_{n}-b_{n} \sin \lambda_{n}
\end{array}\right]=\mathbf{0}
$$

where $\mathbf{q}^{*}$ is now the extended vector $\mathbf{q}^{*} \equiv\left[\begin{array}{ll}\mathbf{q}^{T} & \lambda^{T}\end{array}\right]^{T}$, $\lambda \equiv\left[\begin{array}{llll}\lambda_{1} & \lambda_{2} & \ldots & \lambda_{n}\end{array}\right]^{T}$, and $\boldsymbol{\Phi}\left(\mathbf{q}^{*}\right)$ is a $(m+n) \times 1$ constraint vector, where $\operatorname{dim}(\mathbf{k})=m$, such that $m=3$ for spatial and $m=2$ for planar manipulators. The implicit function theorem for $\boldsymbol{\Phi}$ dictates that if the Jacobian $\boldsymbol{\Phi}_{\mathbf{q}^{*}}=\left[\partial \Phi / \partial \mathbf{q}^{*}\right]$ is nonsingular at a point, then there exists a unique solution in a neighborhood of a point within a tolerance. The Jacobian of the constraint function is

$$
\boldsymbol{\Phi}_{\mathbf{q}^{*}}=\left[\begin{array}{c:c}
\mathbf{k}_{\mathbf{q}} & \mathbf{0} \\
\hdashline \mathbf{I} & \mathbf{q}_{\lambda}
\end{array}\right]
$$

where $x_{q_{i}}=\partial x / \partial q_{i}$ for $i=1, \ldots, n, \quad \mathbf{k}_{\mathbf{q}}=\partial \mathbf{k} / \partial \mathbf{q}$ is a $m \times n$ block submatrix, $\mathbf{q}_{\lambda}=\partial \mathbf{q} / \partial \boldsymbol{\lambda}$ is a $n \times n$ block submatrix, $\mathbf{I}$ is $n \times n$ identity matrix, and $\mathbf{0}$ is a $m \times n$ zero matrix. End-effector velocities can be determined using the analytic Jacobian from

$$
\dot{\mathbf{k}}=\left[\begin{array}{lll}
\dot{x} & \dot{y} & \dot{z}
\end{array}\right]^{T}=\mathbf{k}_{\mathbf{q}} \mathbf{q}_{\boldsymbol{\lambda}} \dot{\boldsymbol{\lambda}}
$$

where $\dot{\lambda}$ is $(n \times 1)$. Define the Null space of $\boldsymbol{\Phi}_{\mathbf{q}^{*}}^{T}\left(\mathbf{q}^{*}\right)$ as the subspace that does not produce any end-effector velocity for a given manipulator configuration. A singular surface is identified in the workspace by a row-rank deficiency condition as

$$
\begin{array}{r}
\partial W \subset\left\{\operatorname{Rank}\left[\boldsymbol{\Phi}_{\mathbf{q}^{*}}\left(\mathbf{q}^{*}\right)\right] \leq(n+m-1),\right. \\
\text { for some } \left.\mathbf{q}^{*} \text { with } \boldsymbol{\Phi}\left(\mathbf{q}^{*}\right)=\mathbf{0}\right\}
\end{array}
$$

The set $\partial W$ identifies singular behavior of the manipulator.

\section{RANK DEFICIENCY OF THE JACOBIAN}

Using the Implicit Function Theorem, and for an $n-\mathrm{DOF}$ system, the Jacobian $\boldsymbol{\Phi}_{\mathbf{q}^{*}}$ is row-rank deficient if and only if one of the following conditions are satisfied.

Condition (i) The upper left $(m \times n)$ corner matrix of $\Phi_{\mathbf{q}}$ is row rank deficient at least one. The rank of the $(m \times n)$ matrix is defined to be the order of the largest non-singular square submatrix that can be formed by selecting rows and columns of the upper corner matrix $\mathbf{k}_{\mathbf{q}}$. Equating the determinants to zero yields $\eta$-number of equations to be solved simultaneously. The solutions to these $\eta$ equations are the singular sets.

Condition (ii) If the matrix $\mathbf{q}_{\lambda}$ is row-rank deficient (i.e., $b_{i} \cos \lambda_{i}$ is zero for either $i=1, \ldots, n$ then $q_{i}$ has reached a limit and the corresponding $\mathbf{k}_{\mathbf{q}}$ is studied for row-rank deficiency. Therefore, the corresponding $\frac{\partial}{\partial \mathbf{q}}\left[\boldsymbol{\Phi}\left(q_{i}, \mathbf{q}\right)\right]$ is subjected to the rank-deficiency conditions. For example, if $q_{i}=q_{i}^{\min }$ (or $q_{i}^{\max }$ ), the diagonal element of $\mathbf{q}_{\lambda}$ will be zero. Thus, the row-rank deficiency of $\Phi_{\mathbf{q}}$ depends on the following submatrix 


$$
\mathbf{k}_{\mathbf{q}} \sim\left[\begin{array}{ccccc}
\mathbf{k}_{q_{1}} & \ldots & \mathbf{k}_{q_{i}} & \ldots & \mathbf{k}_{q_{n}} \\
0 & \ldots & 1 & \ldots & 0
\end{array}\right]
$$

which is a $((m+1) \times n)$ matrix. The solution to this submatrix rank deficiency condition is equivalent to solving the rank deficiency for $\left[\mathbf{k}_{\mathbf{q}} \not \subset \mathbf{k}_{q_{i}}\right]$, with $q_{i}=q_{i}^{\text {limit }}$, where the notation of $\not \subset$ represents the exclusion of the right column from the left matrix. Sub-jacobians are evaluated and their determinants are set to zero. Two generalized coordinates that have reached their limits are identified and denoted by $\mathbf{p}_{i}$ characterized by

$\mathbf{p}_{i}=\left\{\left[\begin{array}{c}\operatorname{det}\left(\hbar_{i} \hbar_{j} \hbar_{k}\right)_{1} \\ : \\ \operatorname{det}\left(\hbar_{i} \hbar_{j} \hbar_{k}\right)_{\eta}\end{array}\right]=\mathbf{0}\right.$, for $i, j, k=1, \ldots, n$ and $\left.i \neq j \neq k\right\}$ $i=1,2, \ldots, \beta$

where $\hbar_{i}$ denotes a column of the matrix $\mathbf{k}_{\mathbf{q}}=\left[\hbar_{1} \ldots \hbar_{n}\right], \beta$ is the total number of singular sets, and $\mathbf{p}_{i}$ is a subset of $\mathbf{q}$ such that $\mathbf{q}^{T}=\left[\begin{array}{ll}\mathbf{p}_{i}^{T} & \mathbf{s}^{T}\end{array}\right]$, i.e., $\mathbf{s}$ contains the remaining $q$ 's.

It is noted that some singularities may be identical. Substituting the set $\mathbf{p}_{i}$ into $\mathbf{k}\left(\mathbf{q}^{*}\right)$ yields a parametric geometric entity (i.e., an equation of a geometric entity). Depending on the number of DOF, the substitution yields a singular surface denoted by

$\boldsymbol{\Gamma}(\mathbf{s})=\mathbf{k}\left(q_{1}^{c}, \ldots, q_{i-1}^{c}, q_{i+1}^{c}, \ldots, q_{j-1}^{c}, q_{j+1}^{c}, \ldots, q_{n}^{c}\right)$

where the superscript $c$ denotes a constant where $\mathbf{s}=\left[\begin{array}{ll}q_{i} & q_{j}\end{array}\right]^{T}$. It was shown by Abdel-Malek and Yeh (1998) that these entities envelop the workspace where perturbation and continuation methods methods were introduced to identify the boundary.

\section{MOTION IN THE NORMAL DIRECTION}

We investigate the admissible normal motion of the endeffector while positioned at a singular entity. At a specific singular configuration of the manipulator $\mathbf{q}_{o}$, the velocity of the end-effector is given by

$$
\dot{k}_{i}=\sum_{j=1}^{n}\left(\frac{\partial k_{i}}{\partial q_{j}}\right)\left(\frac{\partial q_{j}}{\partial \lambda_{j}}\right) \dot{\lambda}_{j}
$$

and in matrix form

$$
\dot{\mathbf{k}}=\mathbf{k}_{\mathbf{q}} \mathbf{q}_{\lambda} \dot{\boldsymbol{\lambda}}
$$

Due to the implicit function theorem and on a singular surface, the term $\left.\mathbf{k}_{\mathbf{q}} \mathbf{q}_{\lambda}\right|_{\mathbf{q}_{o}, \lambda_{o}}$ is rank-deficient. Multiplying both sides by $\boldsymbol{\xi}_{o}^{T}$ (the basis of the null space of $\mathbf{k}_{\mathbf{q}} \mathbf{q}_{\boldsymbol{\lambda}}$ ) yields

$$
\boldsymbol{\xi}_{o}^{T} \dot{\mathbf{k}}=\boldsymbol{\xi}_{o}^{T} \mathbf{k}_{\mathbf{q}}\left(\mathbf{q}_{o}\right) \mathbf{q}_{\lambda} \dot{\boldsymbol{\lambda}}=0
$$

Since $\xi_{o}^{T}$ is a constant vector at $\mathbf{q}_{o}$, the left hand side of Eq. (11) characterizes the equation of a plane in $\mathfrak{R}^{3}$ as $\gamma_{1} \dot{x}+\gamma_{2} \dot{y}+\gamma_{3} \dot{z}=0 \quad$ where $\xi_{o}=\left[\begin{array}{lll}\gamma_{1} & \gamma_{2} & \gamma_{3}\end{array}\right]^{T}$ is indeed a vector normal to the tangent plane of the singular surface at $\mathbf{q}_{o}$. Indeed, for any value of $\dot{\mathbf{q}}$, all resulting velocity vectors $\mathbf{k}$ will lie on a plane which has $\boldsymbol{\xi}_{o}^{T}$ as its normal. Two observations can be made: (i)The basis of the null space of $\left[\mathbf{k}_{\mathbf{q}} \mathbf{q}_{\lambda}\right]^{T}$ is the vector normal to the singular surface (or curve) at $\mathbf{q}_{o}$. (ii) For any given joint velocity vector $\dot{\mathbf{q}}$, the velocity of the end-effector is either tangent to the singular surface or zero, which indicates that the normal component of the end-effector velocity is always zero, i.e.,

$$
v_{n}=\boldsymbol{\xi}_{o}^{T} \dot{\mathbf{k}}=0
$$

Consider the acceleration of a point on the end-effector at a configuration $\mathbf{q}_{o}$ which can be obtained by differentiating Eq. (9) with respect to time and written in index notation $\ddot{k}_{i}=\sum_{j=1}^{n}\left\{\left[\frac{d}{d t}\left(\frac{\partial k_{i}}{\partial q_{j}}\right)\right]\left(\frac{d q_{j}}{d \lambda_{j}}\right) \dot{\lambda}_{j}+\left(\frac{\partial k_{i}}{\partial q_{j}}\right)\left[\frac{d}{d t}\left(\frac{d q_{j}}{d \lambda_{j}}\right)\right] \dot{\lambda}_{j}+\left(\frac{\partial k_{i}}{\partial q_{j}}\right)\left(\frac{d q_{j}}{d \lambda_{j}}\right) \ddot{\lambda}_{j}\right\}$

where $\frac{d}{d t}\left(\partial k_{i} / \partial q_{j}\right) \equiv\left(\partial^{2} k_{i} / \partial q_{l} \partial q_{j}\right)\left(\partial q_{l} / \partial \lambda_{l}\right) \dot{\lambda}_{l}$

and $\frac{d}{d t}\left(\partial q_{j} / \partial \lambda_{j}\right) \equiv\left(\partial^{2} q_{j} / \partial \lambda_{j} \partial \lambda_{j}\right) \dot{\lambda}_{j}$

Expanding terms in the form of joint rates and collecting on similar terms yields

$\ddot{k}_{i}=\dot{\lambda}_{n}\left\{\frac{d q_{j}}{d \lambda_{n}}\left[\frac{d^{2} k_{i}}{d q_{m} d q_{j}}\right] \frac{d q_{j}}{d \lambda_{k}}+\frac{d k_{i}}{d q_{j}} \frac{d^{2} q_{j}}{d \lambda_{n} d \lambda_{k}}\right\} \dot{\lambda}_{k}+\left[\frac{d k_{i}}{d q_{j}} \frac{d q_{j}}{d \lambda_{k}}\right] \ddot{\lambda}_{k}$

Written in matrix form, the acceleration is

$$
[\ddot{\mathbf{k}}]_{i}=\dot{\boldsymbol{\lambda}}^{T} \mathbf{q}_{\lambda}{ }^{T}\left[k_{i}\right]_{\mathbf{q q}} \mathbf{q}_{\lambda} \dot{\lambda}+\dot{\boldsymbol{\lambda}}^{T} \sum_{i=1}^{n}\left(\frac{d k_{i}}{d q_{i}}\left[q_{i}\right]_{\lambda \lambda}\right) \dot{\boldsymbol{\lambda}}+\left[k_{i}\right]_{\mathbf{q}} \mathbf{q}_{\boldsymbol{\lambda}} \ddot{\boldsymbol{\lambda}}
$$

Multiplying both sides of Eq. (17) by the vector $\boldsymbol{\xi}_{o}^{T}$ (the basis of the null space of $\left[\Phi_{\mathbf{q}} \mathbf{q}_{\lambda}\right]^{T}$ ), the last term of the right-hand side vanishes. The normal component of the acceleration is

$$
a_{n}=\boldsymbol{\xi}_{o}^{T} \ddot{\mathbf{k}}=\dot{\lambda}^{T}\left[\mathbf{q}_{\boldsymbol{\lambda}}{ }^{T}\left[\boldsymbol{\xi}_{o}^{T} \mathbf{k}\right]_{\mathbf{q q}} \mathbf{q}_{\boldsymbol{\lambda}}+\sum_{i=1}^{n}\left(\frac{d\left(\boldsymbol{\xi}_{o}^{T} \mathbf{k}\right)}{d q_{i}}\left[q_{i}\right]_{\lambda \lambda}\right)\right] \dot{\boldsymbol{\lambda}}
$$

and written as a quadratic form, the normal component is 


$$
a_{n}=\boldsymbol{\xi}_{o}^{T} \dot{\mathbf{k}}=\dot{\boldsymbol{\lambda}}^{T} \mathbf{H}^{*} \dot{\boldsymbol{\lambda}}
$$

which is a quadratic form, where

$$
\mathbf{H}^{*}\left(\mathbf{q}_{o}, \boldsymbol{\lambda}_{o}\right)=\mathbf{q}_{\lambda}{ }^{T}\left[\boldsymbol{\xi}_{o}^{T} \mathbf{k}\right]_{\mathbf{q q}} \mathbf{q}_{\lambda}+\sum_{i=1}^{n} \frac{d\left(\boldsymbol{\xi}_{o}^{T} \mathbf{k}\right)}{d q_{i}} \cdot\left[q_{i}\right]_{\lambda \lambda}
$$

It is important to observe that on a singular surface $\Gamma_{i}$, any joint acceleration $\ddot{\mathbf{q}}$ does not contribute towards the normal component of the end-effector acceleration. Moreover, since the component of the normal end-effector velocity is zero $\left(v_{n}=0\right)$, with nonzero $a_{n}$, the end-effector may have a component along $\boldsymbol{\xi}_{o}^{T}$ consistent with a positive or negative $a_{n}$.

Consider the planar 2-DOF manipulator shown in Fig. 1a. At $\mathrm{s}$ singularity at $q_{2}=\pi$ which characterizes a singular curve $\Psi_{2}$ shown in Fig. 1b. When the end-effector is located at $\Psi_{2}$, both directions of the normal acceleration $\left(a_{n}^{+}\right.$and $a_{n}^{-}$) may be possible depending on the joint velocity vector $\left[\begin{array}{ll}\dot{q}_{1} & \dot{q}_{2}\end{array}\right]^{T}$.

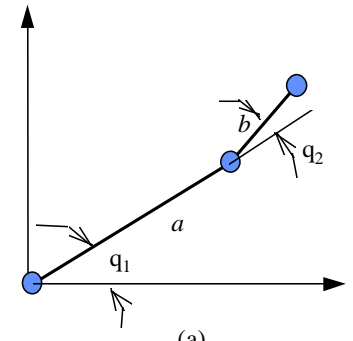

(a)

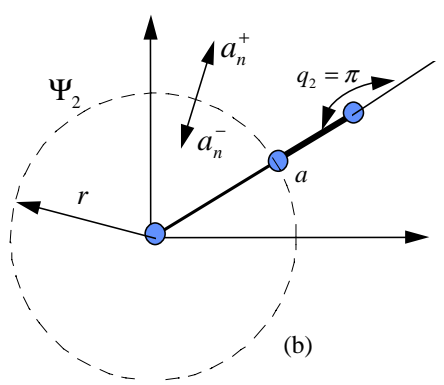

Fig. 1. (a) Planar 2 degree-of-freedom manipulator (b)

Singular curve and normal accelerations

It is clear, however, that the end-effector cannot admit motion towards the inside of $\Psi_{2}$. It admits motion towards the outside of $\Psi_{2}$. The reason is, regardless what values of $\left[\begin{array}{ll}\dot{q}_{1} & \dot{q}_{2}\end{array}\right]^{T}$ are specified, there exists $a_{n}^{-}$(where $a_{n}^{-}$is the component towards the center) that always satisfies

$$
a_{n}^{-}-\frac{v_{t}^{2}}{r} \leq 0
$$

where $v_{t}$ is the velocity of the end-effector tangent to the singular curve and $r$ is the radius of $\Psi_{2}$. This observation leads to a generalization in the following section.

\section{MOTION ALONG THE NORMAL TO A SINGULAR ENTITY}

Consider the end-effector at a point on a singular surface (with radius of curvature $\rho_{o}$ ), the normal acceleration $a_{n}$, and a tangential velocity $v_{t}$. This arm admits motion such that $\mu=a_{n}-\frac{\left|v_{t}^{2}\right|}{\rho_{o}}=\left\{\begin{array}{cc}>0 & \text { moving away from the center } \\ =0 & \text { moving along the singular surface } \\ <0 & \text { moving toward the center }\end{array}\right.$

Note that only using the formulation presented above, it will be possible to determine the expression in Eq. (22) and only because singular surfaces are identified in closed-form.

\section{An expression for $\left(v_{t}^{2} / \rho_{o}\right)$}

For a parametric entity $\Gamma(\mathbf{s}) \in \mathfrak{R}^{3}$, where $\mathbf{s}=\left[\begin{array}{ll}q_{i} & q_{j}\end{array}\right]^{T}$, two fundamental forms are used to characterize a point on the surface. The First Fundamental Form denoted by $\mathbf{I}_{p}$, as

$$
\mathbf{I}_{p} \equiv \delta \mathbf{s}^{T} \Gamma_{\mathbf{s}}^{T} \Gamma_{\mathbf{s}} \delta \mathbf{s}
$$

where $\Gamma_{\mathrm{s}}=\partial \Gamma / \partial \mathrm{s}$ and the Second Fundamental Form as

$$
\mathbf{I I}_{p}=\xi_{o}^{T} \Gamma_{q_{i} q_{i}} d q_{i}^{2}+2 \boldsymbol{\xi}_{o}^{T} \Gamma_{q_{i} q_{j}} d q_{i} d q_{j}+\xi_{o}^{T} \Gamma_{q_{j} q_{j}} d q_{j}^{2}
$$

where the notation $\Gamma_{q_{i} q_{i}}=\partial^{2} \Gamma / \partial q_{i}{ }^{2}, \Gamma_{q_{j} q_{j}}=\partial^{2} \Gamma / \partial q_{j}{ }^{2}$, and $\Gamma_{q_{i} q_{j}}=\partial^{2} \Gamma / \partial q_{i} \partial q_{j}$, and $\boldsymbol{\xi}_{o}^{T}$ is the vector normal to a parametric surface at a point (see Farin 1993 for a complete treatment of parametric surfaces). The Normal Curvature $K_{o}$ of a parametric surface at a configuration $\mathbf{q}_{o}$, in the direction of $d q_{i} / d q_{j}$, can then be defined as the ratio

$$
K_{o}=1 / \rho_{o}=\mathbf{I I}_{p} / \mathbf{I}_{p}
$$

We introduce the Time-Modified First and Second Fundamental Forms as

$$
\begin{aligned}
& \mathbf{I}_{p}^{\prime} \equiv \dot{\mathbf{s}}^{T} \boldsymbol{\Gamma}_{\mathbf{s}}^{T} \boldsymbol{\Gamma}_{\mathbf{s}} \dot{\mathbf{s}} \\
& \mathbf{I I}_{p}^{\prime} \equiv \dot{\mathbf{s}}^{T}\left[\boldsymbol{\xi}^{T} \boldsymbol{\Gamma}\right]_{\mathrm{s}} \dot{\mathbf{s}}
\end{aligned}
$$

respectively, such that the normal curvature can still be defined as

$$
K_{o}=1 / \rho_{o}=\mathbf{I I}_{p}^{\prime} / \mathbf{I}_{p}^{\prime}
$$

In general, the tangential velocity in terms of $\Gamma$ or $\mathbf{k}$ at any point on the surface is

$$
\mathbf{v}_{t}=\Gamma_{\mathbf{s}} \dot{\mathbf{s}}=\mathbf{k}_{\mathbf{q}} \mathbf{q}_{\lambda} \dot{\boldsymbol{\lambda}}
$$

where $\dot{\mathbf{q}}=\mathbf{q}_{\lambda} \dot{\boldsymbol{\lambda}}$. The squared norm of the velocity is

$$
\left|\mathbf{v}_{t}\right|^{2}=\mathbf{v}_{t}^{T} \mathbf{v}_{t}=\dot{\mathbf{s}}^{T} \Gamma_{\mathbf{s}}^{T} \Gamma_{\mathrm{s}} \dot{\mathbf{s}}
$$


which is equal to the Modified First Fundamental Form $\mathbf{I}_{p}^{\prime}$ of Eq. (26). Therefore, $\mathbf{I}_{p}^{\prime}$ can be written as $\quad \mathbf{I}_{p}^{\prime}=\left|v_{t}\right|^{2}$ Substituting for $1 / \rho_{o}$ into $\eta$ yields

$$
\mu=a_{n}-\left|v_{t}\right|^{2} \frac{\mathbf{I I}_{p}^{\prime}}{\mathbf{I}_{p}^{\prime}}
$$

Using Eq. (31), the indicator reduces to

$$
\mu=a_{n}-\mathbf{I I}_{p}^{\prime}
$$

which suggests that

$$
\left|v_{t}^{2}\right| / \rho_{o}=\mathbf{I I}_{p}^{\prime}
$$

The objective is to derive an expression for $\mu$ for any singular surface at a specified point.

\section{AN EXPRESSION FOR THE TIME-MODIFIED SECOND FUNDAMENTAL FORM}

Since $a_{n}$ is in terms of $\dot{\lambda}$ and to express $\mathbf{I I}_{p}^{\prime}$ in terms of $\dot{\lambda}$, the velocity vector on a singular surface is extracted from Eq. (29). Since $\Gamma_{s}$ is not square, a generalized inverse for $\Gamma_{s}$ must be developed. Let $\mathbf{B}$ be a generalized inverse for $\Gamma_{\mathrm{s}}$ such that

$$
\mathbf{B}=\left[\mathbf{E} \Gamma_{\mathrm{s}}\right]^{-1} \mathbf{E}
$$

where $\mathbf{E}$ is a $(2 \times 3)$ matrix defined by $\mathbf{E}=\left[\begin{array}{lll}1 & 0 & 0 \\ 0 & 1 & 0\end{array}\right]$ if the first and second rows of $\Gamma_{\mathbf{s}}$ are independent $\mathbf{E}=\left[\begin{array}{lll}1 & 0 & 0 \\ 0 & 0 & 1\end{array}\right]$ if the first and third rows of $\Gamma_{\mathrm{s}}$ are independent $\mathbf{E}=\left[\begin{array}{lll}0 & 1 & 0 \\ 0 & 0 & 1\end{array}\right]$

if the second and third rows of $\Gamma_{\mathrm{s}}$ are independent such that $\left[\mathbf{E} \boldsymbol{\Gamma}_{\mathrm{s}}\right]$ will become a $(2 \times 2)$ non-singular matrix. Premultiplying Eq. (29) by the generalized inverse yields

$$
\dot{\mathbf{s}}=\left[\mathbf{E} \Gamma_{\mathrm{s}}\right]^{-1} \mathbf{E k}_{\mathrm{q}} \mathbf{q}_{\lambda} \dot{\boldsymbol{\lambda}}=\mathbf{B k}_{\mathrm{q}} \mathbf{q}_{\lambda} \dot{\boldsymbol{\lambda}}
$$

Substituting for $\dot{\mathbf{S}}$ into Eq. (27) yields

$$
\mathbf{I I}_{p}^{\prime}=\dot{\boldsymbol{\lambda}}^{T}\left(\mathbf{q}_{\boldsymbol{\lambda}}\right)^{T}\left(\mathbf{k}_{\mathbf{q}}\right)^{T} \mathbf{B}^{T}\left[\boldsymbol{\xi}^{T} \Gamma\right]_{\mathbf{s s}} \mathbf{B} \mathbf{k}_{\mathbf{q}} \mathbf{q}_{\boldsymbol{\lambda}} \dot{\boldsymbol{\lambda}} \text { (37) }
$$

Substituting $a_{n}$ (Eq. 19) and $\mathbf{I I}_{p}^{\prime}$ (Eq. 37 ) into Eq. (33) yields

$a_{n}-\frac{\left|v_{t}\right|^{2}}{\rho_{o}}=\dot{\boldsymbol{\lambda}}^{T}\left[\mathbf{H}^{*}-\left(\mathbf{q}_{\lambda}\right)^{T} \mathbf{k}_{\mathbf{q}}^{T} \mathbf{B}^{T}\left[\boldsymbol{\xi}_{o}^{T} \Gamma\right]_{\mathrm{ss}} \mathbf{B} \mathbf{k}_{\mathbf{q}} \mathbf{q}_{\lambda}\right] \dot{\boldsymbol{\lambda}}$

If joint limits are considered, the above equation can be written in terms of the new generalized variables as

$$
a_{n}-\mathbf{I I}_{\mathbf{p}}^{\prime}=\dot{\boldsymbol{\lambda}}^{T} \mathbf{Q}^{*} \dot{\boldsymbol{\lambda}}
$$

where

$$
\mathbf{Q}^{*}=\mathbf{H}^{*}-\left(\mathbf{q}_{\lambda}\right)^{T} \mathbf{k}_{\mathbf{q}}^{T} \mathbf{B}^{T}\left[\boldsymbol{\xi}_{o}^{T} \Gamma_{(3]_{s \mathbf{s}}} \mathbf{B} \mathbf{k}_{\mathbf{q}} \mathbf{q}_{\lambda}\right.
$$

which represents a quadratic form.

Parameter at a Limit: In the case of a parameter at its limit, Eq. (39) yields a semi-definite quadratic form (imposed zero velocities). For this case, we propose the projection of a variational movement $\delta \mathbf{k}=\mathbf{k}_{q_{i}} \delta q_{i}$ due to $\delta q_{i}$ onto the normal direction $\xi_{o}^{T}$ such that the normal component is given by

$$
\sigma=\xi_{o}^{T} \mathbf{k}_{q_{i}} \delta q_{i}
$$

where $\delta q_{i}$ is (+1) if $q_{i}$ is at a lower bound and (-1) if at an upper bound. Positive values of $\sigma$ indicate that the entity admita motion in the positive direction of $\boldsymbol{\xi}_{o}^{T}$.

Lemma 1 (crossing a barrier): For a singular configuration $\mathbf{q}_{o}$, which makes the Jacobian $\boldsymbol{\Phi}_{\mathbf{q}}$ row rank deficient, the end-effector is located on a singular surface $\Gamma$. The crossability of a barrier is characterized by the following criteria.

(i) If $\mathbf{Q}^{*}$ is definite, i.e., has both positive and negative eigenvalues, the end-effector can admit normal movements along either direction of $\boldsymbol{\xi}_{o}$. The endeffector can cross this barrier.

(ii) If $\mathbf{Q}^{*}$ is negative or positive semi-definite, the value for $\sigma$ must be computed. If $\sigma$ has a different sign than the nonzero eigenvalues of $\mathbf{Q}^{*}$, the singular surface is crossable. If the all values of $\sigma$ for joints at their limits have the same sign as the nonzero eigenvalues of $\mathbf{Q}^{*}$, the singular surface is non-crossable. The admissible normal direction is determined by this common sign.

\section{Lemma 2 (Void Criterion)}

An enclosure in the workspace of a spatial manipulator or an area in the workspace of a planar manipulator that is bound by geometric entities that do not admit motion towards this enclosure or region is defined as a void. This also includes the definition of the external boundary as it will also exhibit a connected region or surfaces that do not admit motion towards the external. 


\section{A 4R-MANIPULATOR}

Consider the 4R serial manipulator shown in Fig. 2 with joint constraints as follows $-\pi / 2 \leq q_{4} \leq \pi / 2,-\pi \leq q_{3} \leq \pi / 6$, $0 \leq q_{2} \leq 330^{\circ}$, and $0 \leq q_{1} \leq 210^{\circ}$.

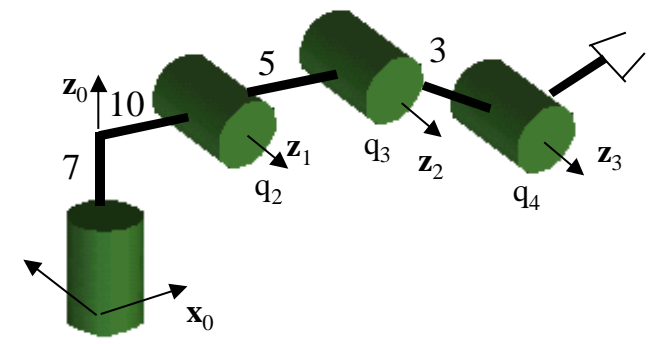

Fig. 2 A 4R manipulator

The position vector function characterizing its workspace is given by

$\mathbf{k}(\mathbf{q})=\left[\begin{array}{c}\cos q_{1}\left(10+5 \cos q_{2}+3 \cos \left(q_{2}+q_{3}\right)+\cos \left(q_{2}+q_{3}+q_{4}\right)\right) \\ \sin q_{1}\left(10+5 \cos q_{2}+3 \cos \left(q_{2}+q_{3}\right)+\cos \left(q_{2}+q_{3}+q_{4}\right)\right) \\ 7+5 \sin q_{2}+3 \sin \left(q_{2}+q_{3}\right)+\sin \left(q_{2}+q_{3}+q_{4}\right)\end{array}\right]$

Using the criteria presented in Abdel-Malek and Yeh (1997), Jacobian rank-deficiency conditions resulting from the row rank deficiency of $\left[\Phi_{\mathbf{q}}\right]^{T}$ and from the reduced-order set yield singular sets. Substituting these singular sets into Eq. (42) yields parametric equations of singular surfaces shown in Fig. $3 \mathrm{a}$, and its cross section in Fig. $3 \mathrm{~b}$.

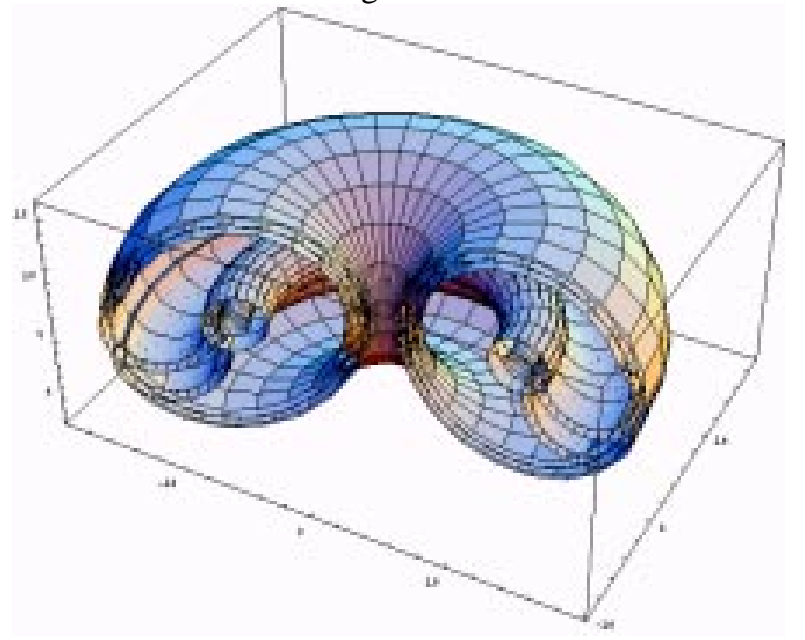

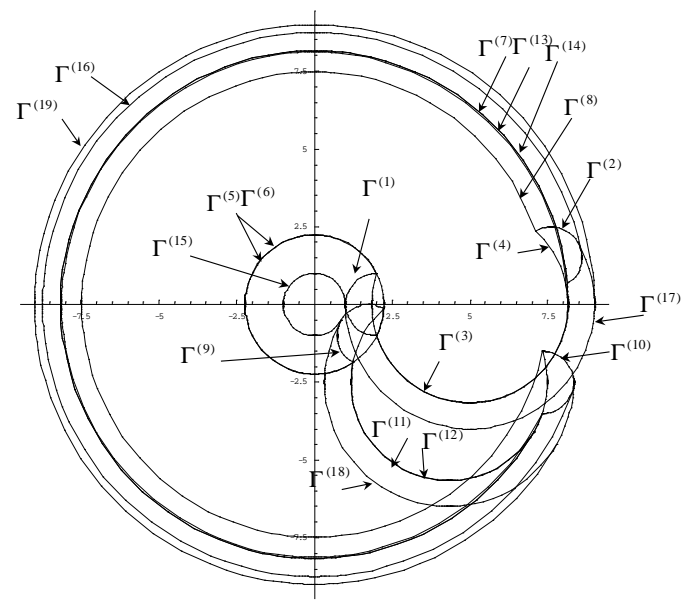

Fig. 3 (a) The workspace depicting all singular surfaces (b) A cross section of the workspace

To determine any voids, it is necessary to evaluate the criteria above for all surface patches. To illustrate the formulation, we shall demonstrate the algorithm for a the point $\mathbf{p}_{o}$ shown in Fig. 4 on surface $\boldsymbol{\Gamma}^{(15)}$, for which the singular set is $\mathbf{p}^{(15)}=\left\{q_{3}=-\pi, q_{4}=0\right\}$, and the point is evaluated at $\mathbf{s}_{o}^{(15)}=\left\{q_{1}=0, q_{2}=200^{\circ}\right\}, \quad$ hence $\quad \mathbf{q}_{o}^{(15)}=\left[\begin{array}{llll}0 & 200^{\circ} & -180^{\circ} & 0\end{array}\right]^{T}$. Substituting $\quad \mathbf{p}^{(15)} \quad$ into Eq. (33) yields $\Gamma^{(15)}\left(\mathbf{u}^{(15)}\right)=\left[\begin{array}{lll}10 \cos q_{1}+\cos q_{1} \cos q_{2} & 10 \sin q_{1}+\cos q_{2} \sin q_{1} \quad 7+\sin \end{array}\right.$

To compute the normal at $\mathbf{p}_{o}$, the matrix $\left[\mathbf{k}_{\mathbf{q}}\left(\mathbf{q}_{o}^{(15)}\right) \mathbf{q}_{\mathbf{s}}\left(\mathbf{s}_{o}^{(15)}\right)\right]^{T}=\left[\begin{array}{ccc}0 & 0 & 0 \\ 0.962 & 0 & -2.644 \\ 0 & 0 & 0 \\ -.0537 & 0 & 1.476\end{array}\right]$ is computed, and its nullspace is readily determined as $\left\{\begin{array}{lll}0.939 & 0 & 0.342\end{array}\right\},\left\{\begin{array}{lll}0 & 1 & 0\end{array}\right\}, \quad$ Since the second vector is trivial, the normal is indeed $\boldsymbol{\xi}_{o}^{T}=\left[\begin{array}{lll}0.939 & 0 & 0.342\end{array}\right] . \quad$ The matrix $\mathbf{H}$ is calculated as $\mathbf{H}=\left[\begin{array}{cccc}0 & 0 & 0 & 0 \\ 0 & 7.920 & 0 & -4.421 \\ 0 & 0 & 0 & 0 \\ 0 & -4.421 & 0 & -2.467\end{array}\right]$ and the generalized inverse is calculated as $\quad \mathbf{B}=\left[\mathbf{E}_{1} \boldsymbol{\Gamma}_{\mathbf{s}}\left(\mathbf{s}_{o}^{(15)}\right)\right]^{-1} \cdot \mathbf{E}_{1}=\left[\begin{array}{ccc}0 & 0.110 & 0 \\ 2.924 & 0 & 0\end{array}\right]$, where the matrix was used. The matrix is computed as and. The final $(4 \times 4) \mathbf{Q}^{*}$ matrix is computed and its eigenvalues are computed as $\{-4.935,0,0,0\}$. Since this is a negative semi definite form, the algorithm must compute a 
value for $\sigma$. Since $q_{3}=-\pi$ is a lower limit, $\delta q_{3}=+1$ and $\sigma=\boldsymbol{\xi}_{o}^{T} \mathbf{k}_{q_{3}}(+1)=0$. Since the normal is along the same direction as the derivative, a value for $\bar{K}$ must be computed. The terms are evaluated as follows: $\left[\boldsymbol{\xi}_{o}^{T} \Gamma\right]_{q_{3} q_{3}}=-4.0$, $\mathbf{k}_{q_{3}}^{T} \mathbf{B}^{T}=\left[\begin{array}{ll}0 & -4\end{array}\right], \quad$ and $\quad\left[\boldsymbol{\xi}_{o}^{T} \Gamma\right]_{\mathbf{u u}} \mathbf{B} \mathbf{k}_{q_{3}}=\left[\begin{array}{ll}0 & -4\end{array}\right]^{T}$. Therefore, $\bar{K}=-20.0056$. A negative sign indicates that the singular surface admits movement into the negative direction of $\boldsymbol{\xi}_{o}^{T}$.

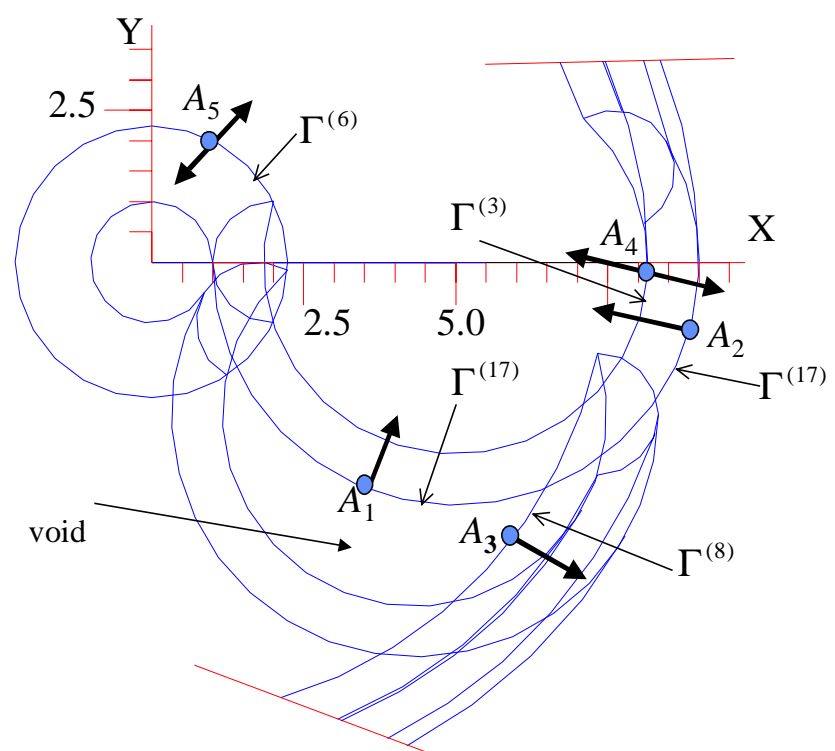

Fig. 4 Part of the swept area showing curve segments

At point $\mathbf{p}_{1}$ on singular surface $\Gamma^{(17)}(\mathbf{u})$ shown on Fig. 4, where $\mathbf{s}^{(17)}=\left\{q_{1}, q_{3}\right\} \quad$ at $\quad \mathbf{p}^{(17)}=\left\{q_{2}^{L}, q_{4}=0\right\} \quad$ and $q_{1}=0, \quad q_{3}=-100^{\circ}$. Evaluating the normal yields $\boldsymbol{\xi}_{o}=\left[\begin{array}{lll}-0.1736 & 0 & -0.9848\end{array}\right]^{T}$. The eigenvalues of $\mathbf{Q}^{*}$ are evaluated $\{-14.1802,-1.8505,0,0\}$, which indicates a negative semi-definite quadratic form. Since $q_{2}$ is at its lower bound $\left(q_{2}^{L}\right)$, the additional value of $\sigma$ is evaluated with $\delta q_{2}=+1$. The value for $\sigma=\xi^{T} \mathbf{k}_{q_{2}} \delta q_{2}=-4.9240$. Since the sign of $\sigma$ is the same as that of the eigenvalues of $\mathbf{Q}^{*}$, it indicates that the surface $\boldsymbol{\Gamma}^{(17)}$ at $\mathbf{p}_{1}$ only admits movement into the opposite direction of the normal $\mathbf{n}_{o}$ (as shown in Fig. 4). This indicates that this surface patch is a boundary.
Similarly, for the same singular surface at point $\mathbf{p}_{2}$ $q_{1}=0, q_{3}=-10^{\circ}$ (which is an external boundary), the normal is $\xi_{o}=\left[\begin{array}{lll}-0.985 & 0 & 0.17\end{array}\right]^{T}$ and the eigenvalues are computed as $\{2.5,1.8505,0,0\}$ and $\sigma=0.868$; same sign as the eigenvalues. This indicates an admissible motion in that direction (but not the opposite direction, i.e., a boundary).

On singular surface $\boldsymbol{\Gamma}^{(8)}$ at point $\mathbf{p}_{3}$ where $\mathbf{s}^{(8)}=\left\{q_{1}, q_{2}\right\}$, and at $\mathbf{p}^{(8)}=\left\{q_{3}^{U}, q_{4}^{U}\right\}$ and $q_{1}=0, q_{2}=305^{\circ}$, the normal is $\xi_{o}=\left[\begin{array}{llll}-0.8032 & 0 & 0.5957\end{array}\right]^{T}$ and the eigenvalues are computed as $\{-2.8974,-1.5389,0,0\}$ and $\sigma=-1.5810$, .

On singular surface $\boldsymbol{\Gamma}^{(3)}$ at point $\mathbf{p}_{4}$ with $\mathbf{s}^{(3)}=\left\{q_{1}, q_{3}\right\}$, at $\mathbf{p}^{(4)}=\left\{q_{2}^{L}, q_{4}^{U}\right\}$ at $q_{1}=0, q_{3}=0^{\circ}$, and $q_{4}=q_{4}^{L}$, the normal is $\xi_{o}=\left[\begin{array}{lll}-0.9487 & 0 & 0.3160\end{array}\right]^{T}$ and the eigenvalues are computed as $\{4.5529,-1.4901,0,0\}$ which is an indefinite quadratic form, i.e., it does not represent a boundary. It is evident that those surface patches that do not admit motion are indeed a boundary. For this example, there are three sets of boundary surface patches shown in Fig. 5 which represent the exact boundary to the workspace including voids.

The external boundary is characterized by the following surface regions

$\Gamma^{(19)}\left(q_{1}, q_{2}\right) ; 0 \leq q_{1} \leq 210^{\circ}$ and $0 \leq q_{2} \leq 330^{\circ}$;

$\Gamma^{(17 c)}\left(q_{1}, q_{3}\right) ; 0 \leq q_{1} \leq 210^{\circ}$ and $34^{\circ} \leq q_{3} \leq 7^{\circ}$,

$\Gamma^{(10)}\left(q_{1}, q_{4}\right) ; 0 \leq q_{1} \leq 210^{\circ}$ and $-20^{\circ} \leq q_{4} \leq 20^{\circ}$

$\Gamma^{(18)}\left(q_{1}, q_{3}\right) ; 0 \leq q_{1} \leq 210^{\circ}$ and $0^{\circ} \leq q_{3} \leq 30^{\circ}$

The inner boundary is characterized by

$\Gamma^{(15)}\left(q_{1}, q_{2}\right) ; 0 \leq q_{1} \leq 210^{\circ}$ and $0^{\circ} \leq q_{2} \leq 330^{\circ}$,

$\Gamma^{(9)}\left(q_{1}, q_{4}\right) ; 0 \leq q_{1} \leq 210^{\circ}$ and $-17^{\circ} \leq q_{4} \leq 0^{\circ}$,

$\Gamma^{(17 b)}\left(q_{1}, q_{3}\right) ; 0 \leq q_{1} \leq 210^{\circ}$ and $-\pi \leq q_{3} \leq-175^{\circ}$.

The boundary to the void is characterized by the following surface

$\Gamma^{(5)}\left(q_{1}, q_{2}\right) ; 0 \leq q_{1} \leq 210^{\circ}$ and $276^{\circ} \leq q_{2} \leq 283^{\circ}$,

$\Gamma^{(17 a)}\left(q_{1}, q_{3}\right) ; 0 \leq q_{1} \leq 210^{\circ}$ and $-154^{\circ} \leq q_{3} \leq-70^{\circ}$, $\Gamma^{(8)}\left(q_{1}, q_{2}\right) ; \quad 0 \leq q_{1} \leq 210^{\circ}$ and $293^{\circ} \leq q_{2} \leq 312^{\circ}, \quad$ and $\Gamma^{(11)}\left(q_{1}, q_{3}\right) ; 0 \leq q_{1} \leq 210^{\circ}$ and $-142^{\circ} \leq q_{3} \leq-30^{\circ}$. patches: 

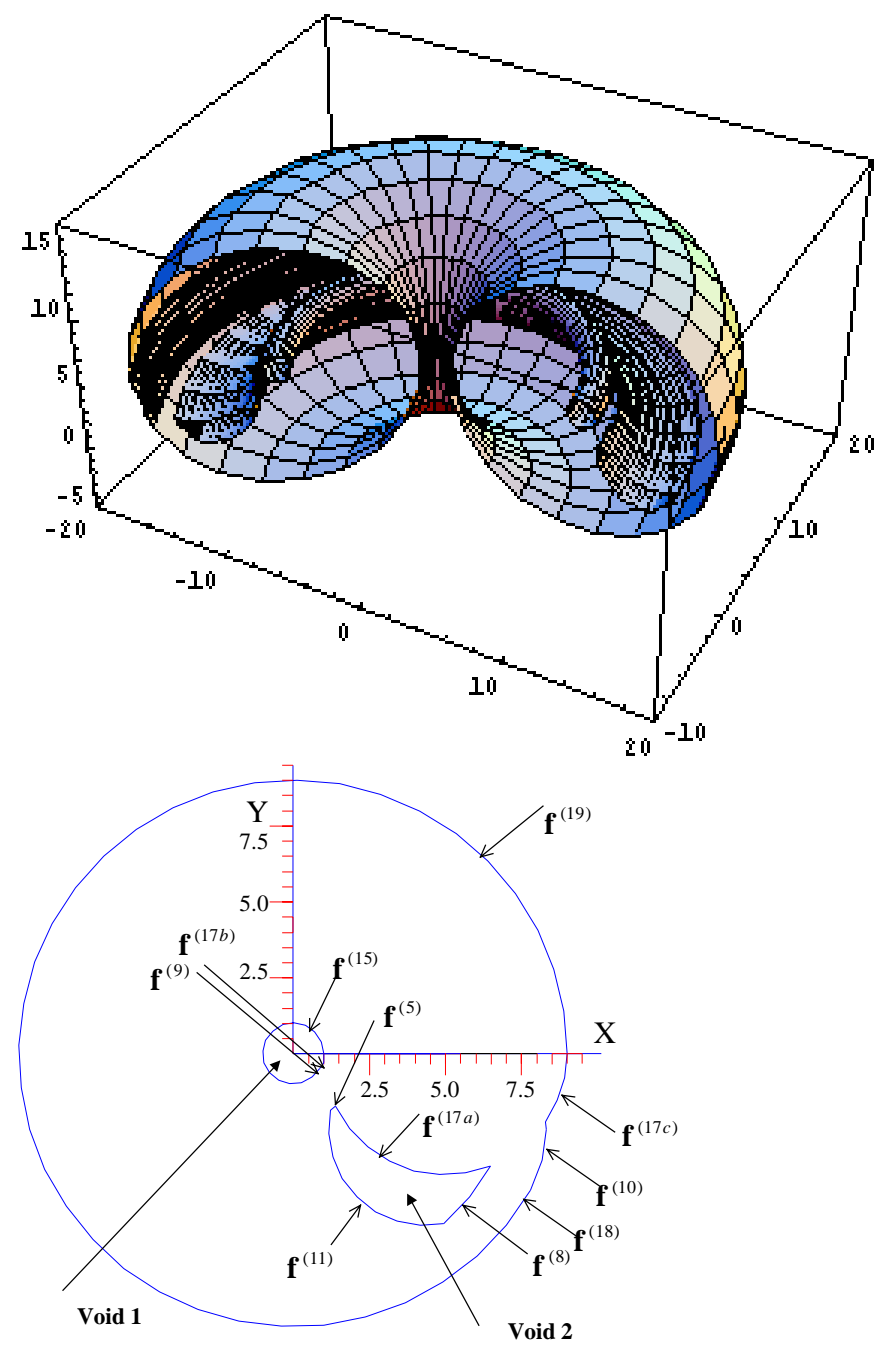

Fig. 5 Identification of boundary curves

\section{A 4DOF RPRP MANIPULATOR}

Consider the manipulator shown in Fig. 6 with two revolute and two prismatic joints. Joint limits are imposed as $0 \leq q_{1} \leq 2 \pi, \quad 20 \leq q_{2} \leq 50, \quad(-\pi / 4) \leq q_{3} \leq \pi, \quad$ and $10 \leq q_{4} \leq 20$.

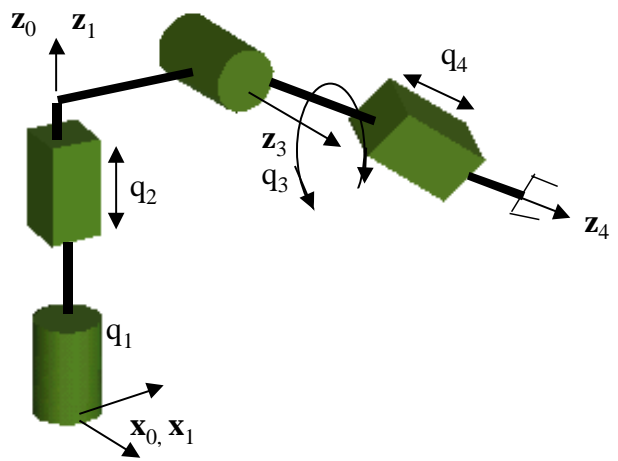

Fig. 6 A four degree-of-freedom manipulator with prismatic joints
For a point on the end-effector, the position vector is given by $\mathbf{k}(\mathbf{q})=\left[q_{4} \cos q_{1} \cos q_{3}+30 \cos q_{1}\right.$

$$
\left.q_{4} \sin q_{1} \cos q_{3}+30 \sin q_{1} \quad q_{4} \sin q_{3}+q_{2}\right]^{T}
$$

Singular sets are entered into Table 1.

Table 1: Singular sets of the spatial manipulator

\begin{tabular}{|l|l|l|l|}
\hline $\mathbf{p}_{1}$ & $q_{3}=0, q_{4}=10$ & $\mathbf{p}_{2}$ & $q_{3}=\pi, q_{4}=10$ \\
\hline $\mathbf{p}_{3}$ & $q_{3}=0, q_{4}=20$ & $\mathbf{p}_{4}$ & $q_{3}=\pi, q_{4}=20$ \\
\hline $\mathbf{p}_{5}$ & $q_{2}=20, q_{3}=-\pi / 4$ & $\mathbf{p}_{6}$ & $q_{2}=20, q_{3}=\pi$ \\
\hline $\mathbf{p}_{7}$ & $q_{2}=20, q_{4}=10$ & $\mathbf{p}_{8}$ & $q_{2}=20, q_{4}=20$ \\
\hline $\mathbf{p}_{9}$ & $q_{2}=50, q_{3}=-\pi / 4$ & $\mathbf{p}_{10}$ & $q_{2}=50, q_{3}=\pi$ \\
\hline $\mathbf{p}_{11}$ & $q_{2}=50, q_{4}=10$ & $\mathbf{p}_{12}$ & $q_{2}=50, q_{4}=20$ \\
\hline $\mathbf{p}_{13}$ & $q_{3}=-\pi / 4, q_{4}=10$ & $\mathbf{p}_{14}$ & $q_{3}=-\pi / 4, q_{4}=20$ \\
\hline
\end{tabular}

Substituting each singularity set into Eq. (35) yields parametric equations of surfaces in $\mathfrak{K}^{3}$ since each equation is parametrized in terms of two variables. Some of these surfaces will indeed act as barriers to output control. Figure 7 is a section of the workspace depicting singular surfaces, and Fig. 7 is a cut at $q_{1}=\pi / 2$ where each surface is identified.

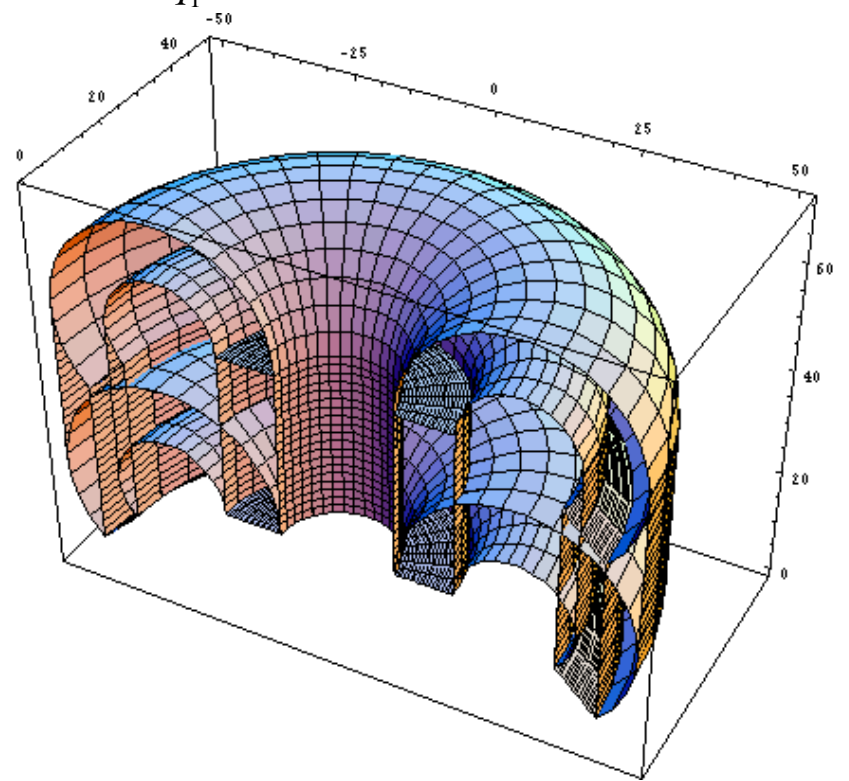

Fig. 7 A section of the workspace depicting singular surfaces

Similar analyses are performed at other points on each region, and those regions that admit output normal movements are indicated as dotted lines in Fig. 8a. 

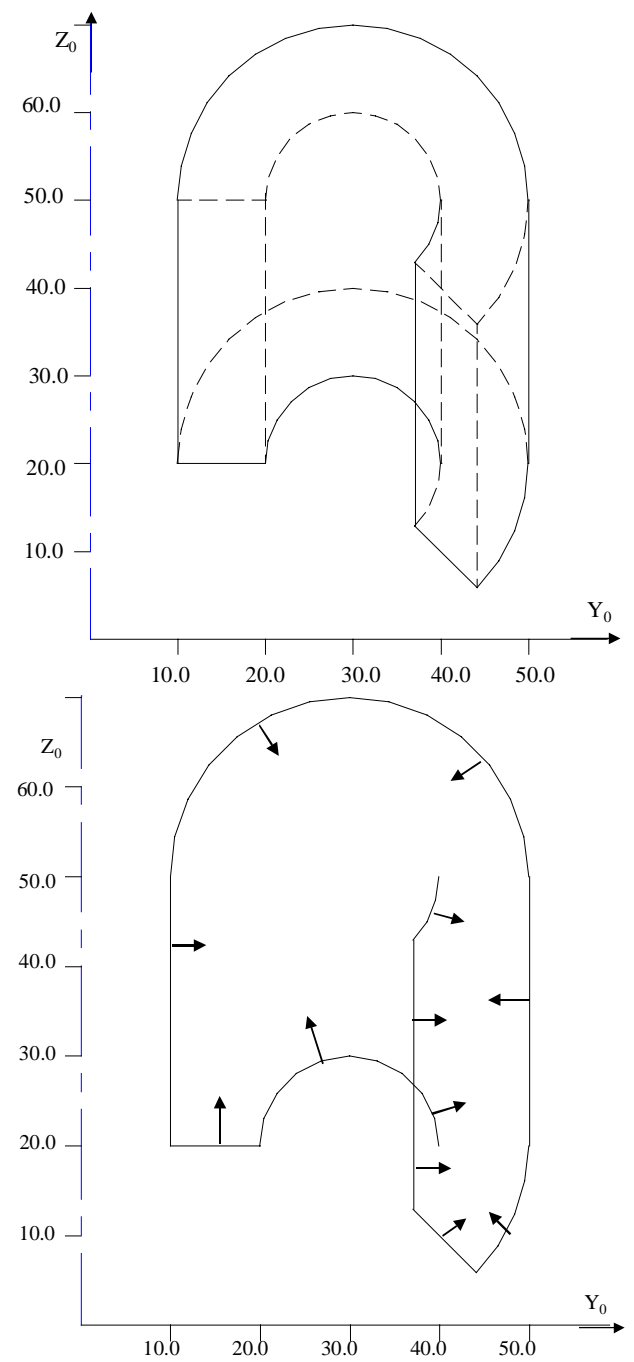

Fig. 8 (a) Admissible output normals (b) a cross-section identifying noncrossable surfaces

Each crossable region of a surface is identified by a dotted line in the cross-section of the workspace shown in Fig. 8a, and only non-crossable regions are shown in Fig. 8b.

\section{CONCLUSIONS}

A formulation for determining output control difficulties and barrier crossability of serial manipulators is presented. Analytic singular entities were determined using conditions imposed on the manipulator Jacobian and on the reduced-order manipulator when a joint constraint is active (joint limits are considered). Three types of singular behavior of a manipulator were identified. The analytic formulation developed in this paper and illustrated using planar and spatial controlled manipulators demonstrates the validity of a general method for determining workspace-internal singular surfaces, their control difficulties, and barrier crossability. It has been shown that all singular entities (curves and surfaces) inside the workspace can be determined.
It was shown that the basis of the null space of the Jacobian at a singular configuration is indeed the normal to a singular surface. This is a significant result that contributes to the understanding of manipulator control behavior at singularities. It also clarifies the significance of a Jacobian singularity with respect to the movement of the manipulator across the surface which was referred to by Oblak and Kohli (1988) as a crossable surface, and more recently by Chevallereau (1996) as feasible trajectory at a singularity.

From the velocity and acceleration analyses, it was shown that the motion across a singular surface is dependent upon the definiteness properties of the Hessian matrix of the basis to the null space, the constraint function, and the fundamental forms of the singular surface. It was also shown that on a singular surface, the joint acceleration vector has no contribution towards the normal component of the end-effector's acceleration.

\section{REFERENCES}

Abdel-Malek, K. and Yeh, H. J., 1997, "On the Determination of Starting Points for Parametric Surface Intersections," Computer-Aided Design, Vol. 29, No. 1, pp.21-35.

Abdel-Malek, K. and Yeh, H.J., 1997, "Analytical Boundary of the Workspace for General Three Degree-of-Freedom Mechanisms," International Journal of Robotics Research. Vol. 16, No. 2, pp. 198-213.

Abdel-Malek, K. and Yeh, H.J., 1996, "Determining Intersection Curves Between Surfaces of Two Solids," Computer-Aided Design, Vol. 28, No. 6-7, pp. 539-549.

Abdel-Malek, K., Adkins, F., Yeh, H.J., and Haug, E.J., 1997, "On the Determination of Boundaries to Manipulator Workspaces," Robotics and Computer-Integrated Manufacturing, Vol. 13, No. 1, pp. 63-72.

Ahmad, S. and Luo, S. 1988. Analysis of kinematic singularities for robot manipulators in Cartesian coordinate parameters. Proceedings of the IEEE Int. Conf. on Rob. and Autom., Philadelphia, PA, 840-845.

Chevallereau, C. 1996. Feasible trajectories for a non redundant robot at a singularity. Proc. of IEEE Int. Conf. on Rob. and Aut., Minneapolis, MN, pp. 1871-1876.

Denavit, J. and Hartenberg, R.S., 1955. A kinematic notation for lower-pair mechanisms based on matrices. Journal of Applied Mechanics, 77:215-221.

Farin, G., Curves and Surfaces for Computer-Aided Geometric Design, Academic Press, London, UK.

Fu, K. S. Gonzalez, R. C. and Lee, C. S. G. 1987. Robotics: Control, Sensing, Vision, and Intelligence, McGraw-Hill, Inc., New York.

Haug, E. J. Adkins, F. A. Qiu, C. C. and Yen, J. 1995. Analysis of barriers to control of manipulators within accessible output sets. Proceedings of the 20th ASME Design 
Engineering Technical Conference, Boston, MA, 82:697704.

Haug, E.J., Luh, C.M., Adkins, F.A., and Wang, 1996, "Numerical algorithms for mapping boundaries of manipulator workspaces," Trans. ASME J. Mech. Des., 118 (1), 228-234

Lai, Z. C. and Yang, D. C. H. 1984. On the singularity analysis of simple six-link manipulators ASME Paper No. 84-DET-220.

Lai, Z. C. and Yang, D. C. H. 1986. A new method for the singularity analysis of simple six-link manipulators. Int. J. of Rob. Res., 5(2):66-74.

Lipkin, H. and Pohl, E. 1991. Enumeration of singular configurations for robotic manipulators. ASME J. Mech., Trans., and Autom. in Des., 113:272-279.

Litvin, F. L. Fanghella, P. Tan, J. and Zhang, Y. 1986a. Singularities in motion and dispplacement functions of spppatial linkages. ASME J. of Mech., Trans., and Autom. in Des., 108(4):516-523.

Litvin, F. L. Yi, Z. Castelli, V. P. and Innocenti, C. 1986 b. Singularities, configurations, and displacement functions for manipulators. Int. J. Rob. Res., 5(2):52-65.

Merlet, J. P. 1989. Singular configurations of parallel manipulators and Grassman geometry. Int. J. of Rob. Res., 8:45-56.

Mohammadi Daniali, H. R., Zsombor-Murray, P. J. and Angeles, J. 1995. Singularity analysis of planar parallel manipulators. Mechanism and Machine Theory, 30:665-678.

Nielsen, L. deWit, C. C. and Hagander, P. 1991. Controllability issues of robots in singular configurations. Proceedings of IEEE Int. Conf. on Rob. and Autom., Sacramento, CA.

Oblak, D. and Kohli, D., 1988. Boundary surfaces, limit surfaces, crossable and noncrossable surfaces in workspace of mechanical manipulators. ASME Journal of Mechanisms, Transmissions, and Automation in Design, (110):389-396.

Pai, D. K. and Leu, M. C. 1992. Generecity and singularities of robot manipulators. IEEE Trans. on Rob. Autom., 8:545559.

Shamir, T. 1990. The singularities of redundant robot arms. Int. J. of Rob. Res., 2(1):113-121.

Shu, M. Kohli, D. and Dwivedi, S. H. 1986. Proceedings of the 6th World Congress on Theory of Machines and Mechanisms, New Delhi, India, 988-993.

Soylu, R. and Duffy, J. 1988. Hypersurfaces of special configurations of serial manipulators and related concepts. Part II: Passive joints, configurations, component manifolds and some applications. J. Rob. Systems, 5:31-53.

Spanos, J. and Kohli, D. 1985 "Workspace Analysis of Regional Sttructure of Manipulators," ASME J. of Mech. Trans. and Aut. in Design, Vol. 107, pp. 219-225.

Tourassis, V. D. and Ang, M. H. 1992. Identification and analysis of robot manipulator singularities. Int. J. of Rob. Res., 11:248-259.
Waldron, K. J. 1987. Operating barriers within the workspace of manipulators. Proceedings of the Society of Manufacturing Engineers, Chicago, IL, Robots II/17th ISIR, 8:35-46.

Waldron, K. J. Wang, S. L. and Bolin, S. L. 1985. A study of the Jacobian matrix of serial manipulators. ASME J. of Mech., Trans., and Autom. in Des., 107(2):230-238.

Wang, S. L. and Waldron, K. J. 1987. Study of the singular configurations of serial manipulators. ASME J. of Mech., Trans., and Autom. in Des., 109(1):14-20.

Yeh, H.J. 1996, Singularity and Workspace Analyses of Serial Robot Manipulators. PhD Thesis, The University of Iowa, Iowa City, IA, December 1996. 